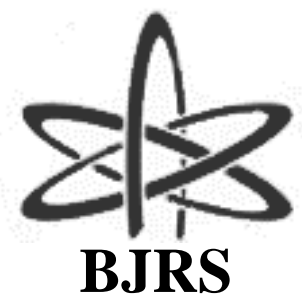

\author{
BRAZILIAN JOURNAL \\ $\mathrm{OF}$ \\ RADIATION SCIENCES \\ 07-02A (2019) 01-15
}

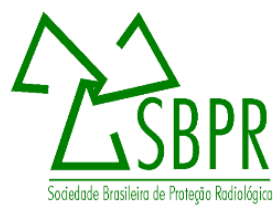

\title{
Proposed Parameters for a Circular Particle Accelerator for Proton Beam Therapy Obtained by Genetic Algorithm
}

\author{
Gustavo Lobato Campos ${ }^{1}$, Tarcísio P. R. Campos ${ }^{2}$ \\ 1-Instituto Federal de Minas Gerais, 2-Universidade Federal de Minas Gerais \\ gustavo.lobato@ifmg.edu.br
}

\begin{abstract}
This paper brings to light optimized proposal for a circular particle accelerator for proton beam therapy purposes (named as ACPT). To obtain optimized parameters of the equipment we applied computational metaheuristics based on genetic algorithms (GA). Some fundamental concepts in the metaheuristics developed in Matlab® software will be presented. Four parameters were considered for the proposed modeling for the equipment, being: potential difference, magnetic field, length and radius of the resonant cavity. As result, this article showed optimized parameters for two ACPT, one of them used for ocular radiation therapy, as well some parameters that will allow teletherapy, called in order ACPT - 65 and ACPT - 250, both of them obtained through metaheuristics based in GA.
\end{abstract}

Keywords: circular particle accelerator, proton therapy, genetic algorithm. 


\section{INTRODUCTION}

\subsection{CONSIDERATIONS FOR A PROTON THERAPY}

Protons, like heavy ions, interact differently with matter when compared to electromagnetic radiation, such as X-Rays and Gamma Rays. The intensity of the beam decays exponentially with depth for the electromagnetic radiation, and thus does not have a wide reach (WIESZCZYKA; SCHARF, 2001). In opposition to the proton beam, as presented by Fig. 1 of (MAGALLANES; HERNÁNDEZ; FERNÁNDEZ, 2015) with the energy deposition curves by photons and protons beams.

Figure 1: Representation of the energy deposition of photon beam and protons.

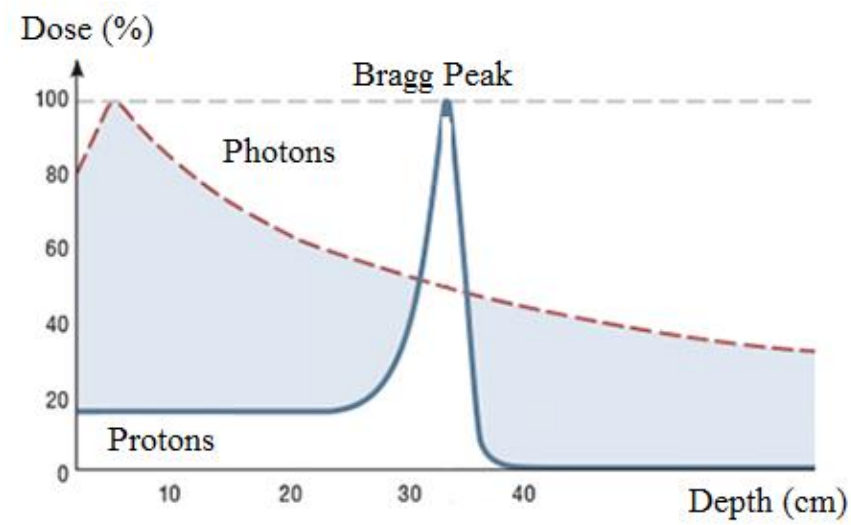

Source: MAGALLANES; HERNÁNDEZ; FERNÁNDEZ, 2015.

According to (WIESZCZYKA; SCHARF, 2001), the use of proton beam therapy has an advantage over X-ray bundles and gamma rays because protons have a higher deposition of ionizing energy in depth with reduced lateral scattering, unlike photons and, therefore, provide a low attack less to healthy tissues posterior and anterior to the tumor. Therefore, the concentration of energy deposition in a specific region (Bragg peak) is one of the most important characteristic of proton beam.

During its course through matter the charged particle ionizes the atoms of the material and deposits some of its energy in the absorbed dose form, with Bragg Peak being the point of maximum deposition, highlighted in Fig.1, which occurs before the particle reaches the rest. In this region, the protons lose most of their kinetic energy and consequently their velocity is reduced to zero, because of Coulomb interaction with the electrons and with the nuclei of the atoms of the material (TUNER, 1992). Another characteristic evidenced by Fig. 1 is the almost constant dose distribution at initial depths, 
which is approximately $20 \%$ to $30 \%$ of the maximum dose, as reported by (NIFENECKER, 1999). According to (WIESZCZYKA; SCHARF, 2001), the deflection of the proton depth-dose curve is very small at first, when compared to the electron curve, mainly due to the difference in mass of the two particles. The occurrence of Bragg Peak is related to the proton interaction shock section that increases as the energy and velocity of the particles decrease, a fact verified at the end of the particle path like showed by (LOMAX, 2003). Thus for application in radiotherapy the protons are usually accelerated to sufficient energies so that the Bragg Peak is present in the tumor region.

\subsection{CONSIDERATIONS ABOUT CIRCULAR ACCELERATORS}

Circular particle accelerators are very important equipment for radiotherapy, since they have the possibility of oriented applications of accelerated particle bundles for the purpose of cancer treatment. In 1950s the first clinical trials in proton therapy, more precisely in 1954 in Berkeley, United States, and in 1957 in Uppsala, Sweden. After these experiments, we observed an increasing number of environments built to attend radiotherapy treatments with protons. It is important to mention the advent of computed tomography in the 1970s, where provide great advances in medical imaging, with anatomical discrimination and precise identification of tumors, favored the use of proton radiation therapy to treat several tumors (WIESZCZYKA; SCHARF, 2001).

The charged particle accelerated by the circular particle accelerator, with determined kinetic energy, has been the basis for proton radiation therapy. Thus, it is necessary to produce a beam of particles with sufficient kinetic energy for penetration into the organs affected by cancer. In this way, it is extremely important to adjust the parameters of a circular particle accelerator to deliver a beam with the necessary characteristics to have adequate penetration and deposit the necessary energy in the tumor.

The model in which metaheuristic was proposed, developed and applied is shown in Fig. 2. The same is based on the work of (RABELO, 2016). The model consists essentially of three regions: the acceleration structure, the particle beam circulation structure, and the spacing region. The acceleration structure is where the energy gradient will be provided to the particle to be accelerated by the application of an electric field, or more specifically due to a potential difference present. Already the circulation structure is that where the particle undergoes a magnetic field action, so that after a complete 
cycle the equipment returns to the region of the acceleration structure. And finally the region of separation where the particle in acceleration will "waste time" assisting in the process of synchronism.

Figure 2: Representation of the circular particle accelerator model for which metaheuristics was developed.

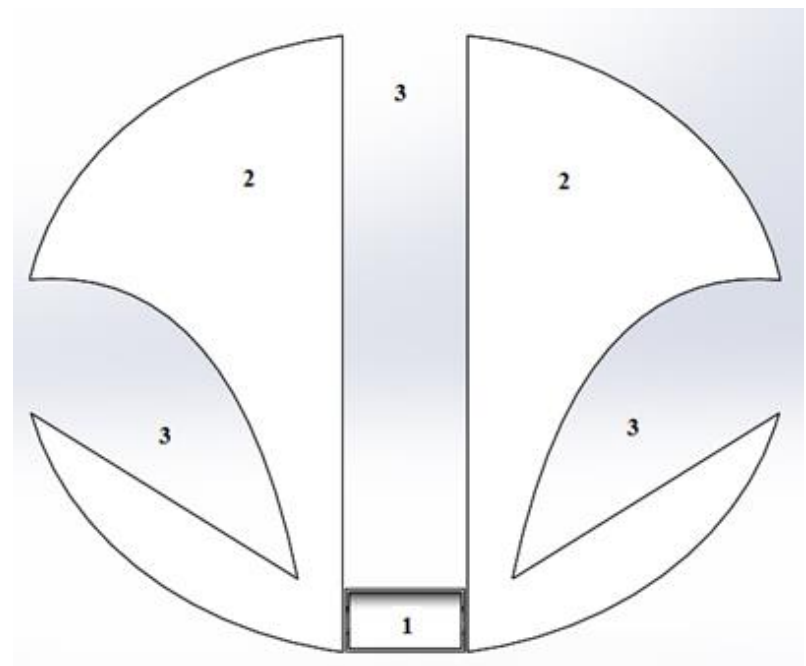

Source: by author.

In summary, this model consists of two structures: the acceleration structure (identified in the figure by 1), specifically a resonant cylindrical cavity, and the circulation structure (identified in the figure by 2). The differential of the equipment against a traditional cyclotron is in its fins or gaps (identified in the figure by 3), where the particle in acceleration process no longer undergoes action of the magnetic field of the circulation region, and thus becomes feasible its synchronism In the acceleration process by passing the acceleration structure several times.

It is important to mention that it is not the focus of this paper to present the mathematical modeling of the cited equipment model.

Therefore, in order to search for the best composition of certain parameters of the circular particle accelerator, a metaheuristic was developed, based on genetic algorithms (GA), implemented in software Matlab®, resulting in parameters such as potential difference, magnetic field, length and radius of the resonant cavity. Thus obtaining optimized essential parameters for modeling circular particle accelerators for proton beam therapy, ACPT.

\section{SOFTWARE DEVELOPMENT, AG - ACPT}




\subsection{THERAPY}

In this paper, the final energy of the proton beam was adopted as two distinct values, as presented in Table 1 . This value is related to the energy whose proton beam penetrates and adequately covers the target anatomical region with the dose prescribed in the treatment.

Table 1: Energies evaluated in this paper, for ocular and body therapy.

\begin{tabular}{|c|c|}
\hline Application & Energy \\
\hline Ocular Therapy & 5 to $65 \mathrm{MeV}$ \\
\hline Body Therapy & 5 to $250 \mathrm{MeV}$ \\
\hline
\end{tabular}

Source: by author.

\subsection{GENETIC ALGORITHM}

Genetic algorithms are possibly one of the main techniques used in the field of evolutionary computation. The origin of GA dates back to the 1960s, more specifically the work of probabilistic algorithms by University of Michigan's professor, John Holland. These works culminated in the publication in 1975 of the book "Adaptation in Natural and Artificial Systems", which presented a scope of action for the GA wider than pure and simple optimization. Already in the 80s, mention is made of David Goldberg's work, with the use of GA in solving optimization problems, especially the book "Genetic Algorithms in Search, Optimization, and Machine Learning".

Thus, today the GA are metaheuristics, which are part of the area of evolutionary computation, being applied in the resolution of several types of optimization problems, and inspired by the mechanism of evolution of the species, as summarized in Fig. 3.

Figure 3: Representation of the development of genetic algorithms.

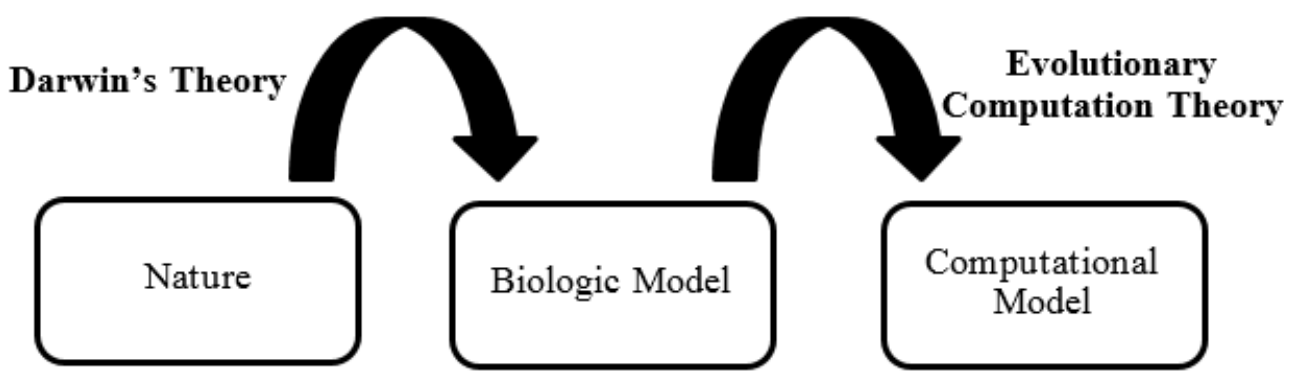

Source: by author. 
A pseudocode of a generic genetic algorithm is depicted in Fig. 4. It should be noted that the metaheuristics, developed and validated as presented in chapter three of this document, is applied at this moment to generate optimized values for parameters of the proposed circular particle accelerators for proton beam therapy.

Figure 4: Genetic algorithm pseudo-code [6].

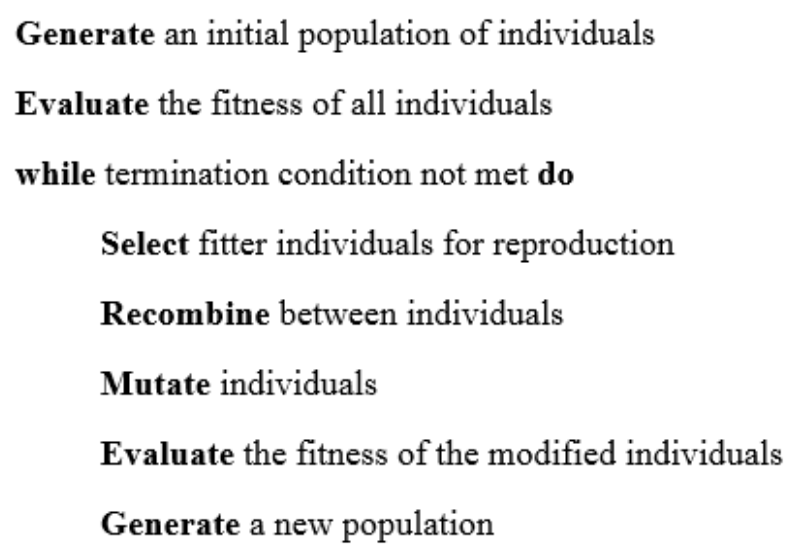

End while

Source: by author.

The main topics associated with the development of GA, as presented in Fig. 4, are described below.

\subsubsection{POPULATION}

The population of a GA refers to the set of individuals that will be evaluated as a possible solution to the problem, just as they will be used to create the new set of individuals for analysis.

Population size can affect the overall performance and efficiency of genetic algorithms. Very small populations have great chances of losing the diversity required to converge to a good solution as they provide a small coverage of the problem search space. However, if the population has many individuals, the algorithm may lose much of its efficiency because of the delay in evaluating the fitness function of the whole set at each iteration (HOLLAND, 1975) and (CARUANA; SCHAFFER, 1988).

\subsubsection{ADAPTATIVE FUNCTION, OR FITNESS}

Through a certain adaptive function or fitness, the fitness value of each individual of the population is specified. It is one of the most important components of any GA, since it is by means of which it 
is quantified how close an individual is to the desired solution or how good each solution is. Therefore, it is fundamental that this function be representative and differentiate in good proportion to good and bad solutions. If the accuracy in the evaluation is not adequate, a good solution can be discarded during the execution of the algorithm, besides being able to take more time to explore less promising solutions (MITCHELL, 1997).

The function of population assessment, or fitness, is shown in Fig. 5. It is noteworthy that it has seven terms, four of them associated to the behavior of the particle per orbit in the equipment, and three other terms associated with constraints to guarantee answers within the valid range for the problem.

Figure 5: Part of the code associated with calculating the fitness of each individual.

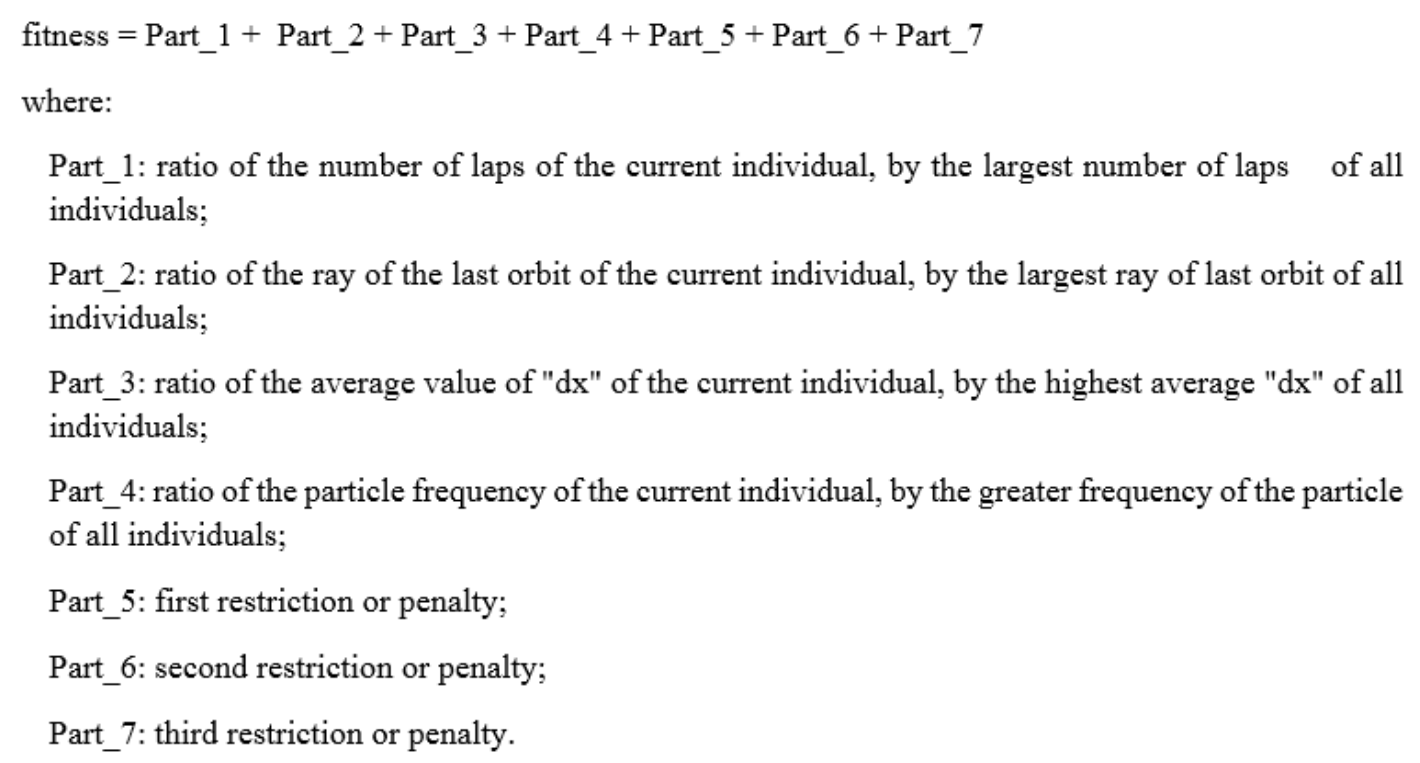

Source: by author.

\subsubsection{SELECTION OF THE POPULATION}

From a population where an aptitude value was assigned to each individual, a method was proposed to select the individuals on which the genetic operators will be applied. Among the various forms or techniques of selection, the methods of selection by Roulette and Tournament are of relevance according to (MITCHELL, 1997).

In Roulette selection, each individual of the population is represented in roulette in proportion to his or her fitness index. Thus, for individuals with high fitness a larger portion of the roulette area is given; while, for individuals of lower fitness, a relatively smaller portion is given. In this method, one 
of the identified problems is associated to the processing time, since the method requires two passages for all the individuals of the population (BANZHAF; NORDIN; KELLER; FRANCONE, 1998). Already in the Tournament selection, we have a number " $n$ " of individuals of the randomly selected population to form a temporary subpopulation. From this group, the selected individual will depend on a previously defined probability "k". This method is usually the most used, for the advantage of not requiring that the comparison be made among all the individuals of the population (BANZHAF; NORDIN; KELLER; FRANCONE, 1998).

\subsubsection{GENETIC OPERATORS}

The search for a satisfactory result, through the transformation of the population through successive generations, is only viable by the genetic operators, responsible for the transformation process of the population. Genetic operators are necessary for the population to diversify, but at the same time maintain adaptation characteristics acquired by previous generations. It stands out in GA the operators of crossing, also known as crossover or recombination and mutation.

- Recombination: it is the predominant genetic operator. Through the recombining are created new individuals blending characteristics of two individuals "parents". This mixture is made $b$ $y$ trying to mimic the reproduction of genes in cells. Excerpts of the characteristics of an indi vidual are exchanged for the equivalent portion of the other. The result of this operation is an individual that potentially combines the best characteristics of the individuals used as a basis (POZO; CAVALHEIRO; ISHIDA; SPINOSA; RODRIGUES, 2016).

- Mutation: this operation randomly modifies some characteristic of the individual upon which it is applied. This exchange is important because it ends up creating new values of characteri stics that did not exist or appeared in small numbers in the population under analysis. The $\mathrm{m}$ utation operator is necessary for the introduction and maintenance of the genetic diversity of the population. In this way, the mutation ensures that the probability of reaching any point in the search space is possibly not zero. The mutation operator is applied to individuals through a generally small mutation rate (POZO; CAVALHEIRO; ISHIDA; SPINOSA; RODRIGUE S, 2016). 


\subsubsection{CONFIGURATION PARAMETERS OF GA}

At each step, a new set of individuals is generated from the previous population. "Generation" is the denomination for this new set. It is observed that with a large number of generations, good results are obtained through the GA (POZO; CAVALHEIRO; ISHIDA; SPINOSA; RODRIGUES, 2016).

\section{COMPUTATIONAL SIMULATION ACPT}

The implemented metaheuristics was applied considering the parameters below for ACPT equipment, with: Number of individuals 150; Number of generations 100; and Total number of rounds 10. Two energy ranges were evaluated to meet the requirements of Table 1. It is also important to mention that the limits for the variables to be adopted by the algorithm are those highlighted in Table 2 .

Table 2: Variables of the population generation function.

\begin{tabular}{|c|c|c|}
\hline Number of individuals - NI & \multicolumn{2}{|c|}{150} \\
\hline Number of variables - NV & \multicolumn{2}{|c|}{4} \\
\hline \multirow{3}{*}{ Low limit - Linf } & ddp & 100 \\
\cline { 2 - 3 } & B & 0.5 \\
\cline { 2 - 3 } & Lcav & 0.1 \\
\cline { 2 - 3 } & Rcav & 0.1 \\
\hline \multirow{3}{*}{ High limit - Lsup } & ddp & 400 \\
\cline { 2 - 3 } & B & 3 \\
\cline { 2 - 3 } & Lcav & 0.6 \\
\cline { 2 - 3 } & Rcav & 0.6 \\
\hline
\end{tabular}

\subsection{OCULAR THERAPY}

The first simulation beyond in ACPT it was to particle beam of protons with initial energy of $5 \mathrm{MeV}$ and final energy of $65 \mathrm{MeV}$.

Therefore, in Table 3 it was present the best solutions for the 10 rounds analyzed by computational routine. Each of the 10 rows represent the best solutions for each of the rounds evaluated. As for the columns, we have the representation of the four parameters: ddp, B, Lcav, Rcav, and finally the fitness function value. 
Table 3: Best solutions for each of the 10 rounds evaluated.

\begin{tabular}{|l|c|c|c|c|c|}
\hline & $\begin{array}{c}\text { Potential diffe- } \\
\text { rence (kV) }\end{array}$ & $\begin{array}{c}\text { Magnetic fi- } \\
\text { eld (T) }\end{array}$ & $\begin{array}{c}\text { Resonant cavity } \\
\text { length (m) }\end{array}$ & $\begin{array}{c}\text { Resonant cavity } \\
\text { radius (m) }\end{array}$ & fitness \\
\hline Round 1 & 327.7206 & 0.8004 & 0.4076 & 0.3453 & 1.7095 \\
\hline Round 2 & 277.4367 & 1.6459 & 0.2223 & 0.1596 & 1.6358 \\
\hline Round 3 & 195.7065 & 0.7149 & 0.301 & 0.2978 & 1.7591 \\
\hline Round 4 & 369.8364 & 1.1601 & 0.1002 & 0.2073 & 1.2755 \\
\hline Round 5 & 338.4495 & 0.9905 & 0.1671 & 0.2607 & 1.4484 \\
\hline Round 6 & 291.2818 & 1.8977 & 0.2736 & 0.1497 & 1.5515 \\
\hline Round 7 & 327.1671 & 0.7709 & 0.1728 & 0.2917 & 1.5170 \\
\hline Round 8 & 257.4707 & 0.6675 & 0.2133 & 0.3911 & 1.6057 \\
\hline Round 9 & 227.5528 & 1.3531 & 0.1115 & 0.2138 & 1.5156 \\
\hline Round 10 & 300.0718 & 0.7076 & 0.1788 & 0.2355 & 1.5980 \\
\hline
\end{tabular}

The best parameter composition presented by the AG - ACPT occurred in the round of number 4, with a fitness value of 1.2755. Also for this round, other important features presented by the algorithm were the particle frequency of $15.8239 \mathrm{MHz}$ as well as the resonance frequency of the cylindrical cavity of 553.8359 MHz. For both frequencies to be multiple, by a factor of 35, it can make possible the presence of multiple bubbles of particles in the equipment.

The evolution of fitness for the best condition presented by the AG - ACPT is shown in Fig. 6. It is verified that the best answer occurred in the generation number 92 .

Figure 6: Evolution of fitness to the next 100 generations for the round that presented the best answer.

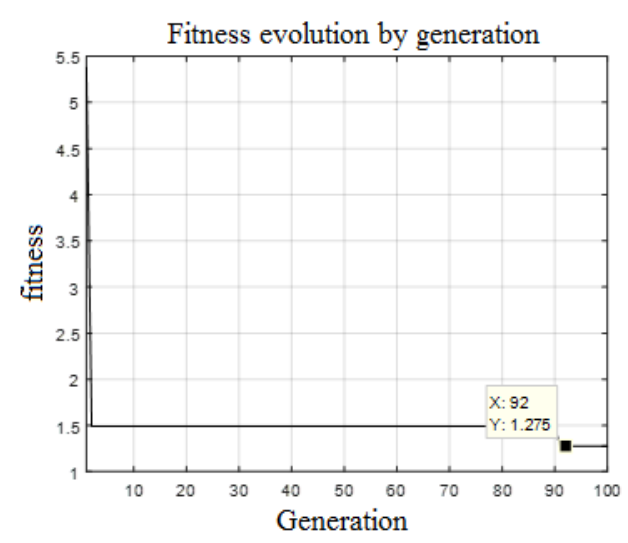

Source: by author. 
The values presented as the best answer by the AG - ACPT, are highlighted in Table 4.

Table 4: Best response for the parameters for the circular particle accelerator according to the metaheuristic ACPT - 65 .

\begin{tabular}{|c|c|}
\hline Potential difference (kV) & 369.8364 \\
\hline Magnetic field (T) & 1.1601 \\
\hline Resonant cavity length (m) & 0.1002 \\
\hline Resonant cavity radius (m) & 0.2073 \\
\hline
\end{tabular}

Based on the optimized physical parameters, the main characteristics for the ACPT - 65 model obtained with the aid of the GA were then evaluated, as shown in Table 5.

Table 5: Features of the ACPT-65.

\begin{tabular}{|c|c|}
\hline \multicolumn{2}{|c|}{ ACPT $-\mathbf{6 5}$} \\
\hline Turns on accelerator & 162 \\
\hline Internal radius (m) & 0.2789 \\
\hline External radius (m) & 1.0207 \\
\hline Maximum Gap (m) & 0.1616 \\
\hline Frequency of accelerator cavity (MHz) & 553.8359 \\
\hline Number of bubbles & 35 \\
\hline Timing period (s) & $6.320 \times 10^{-8}$ \\
\hline
\end{tabular}

\section{2. $250 \mathrm{MeV}$ THERAPY}

For this second simulation, the initial energy value of the proton was maintained at $5 \mathrm{MeV}$, but the final energy was $250 \mathrm{MeV}$, according to Table 6, to meet the proton therapy of the thorax.

Table 6 presents the best solutions for the 10 rounds evaluated by the computational routine. Again, each of the 10 rows represent the best solutions for each of the rounds evaluated. As for the columns, we have the representation of the 4 variables, potential difference, magnetic field, length and radius of the resonant cavity, and lastly the value of the fitness function. 
Table 6: Best solutions for each of the 10 rounds evaluated.

\begin{tabular}{|l|c|c|c|c|c|}
\hline & $\begin{array}{c}\text { Potential diffe- } \\
\text { rence (kV) }\end{array}$ & $\begin{array}{c}\text { Magnetic fi- } \\
\text { eld (T) }\end{array}$ & $\begin{array}{c}\text { Resonant cavity } \\
\text { length (m) }\end{array}$ & $\begin{array}{c}\text { Resonant cavity } \\
\text { radius (m) }\end{array}$ & fitness \\
\hline Round 1 & 339.9669 & 1.7060 & 0.2102 & 0.1356 & 1.3784 \\
\hline Round 2 & 311.4621 & 1.6009 & 0.2544 & 0.1531 & 1.5546 \\
\hline Round 3 & 393.8205 & 0.9969 & 0.3835 & 0.2594 & 1.4764 \\
\hline Round 4 & 243.0381 & 1.8047 & 0.2698 & 0.1714 & 1.7166 \\
\hline Round 5 & 379.7419 & 1.2031 & 0.4028 & 0.2491 & 1.6641 \\
\hline Round 6 & 350.5658 & 0.7976 & 0.4218 & 0.2564 & 1.5278 \\
\hline Round 7 & 255.2408 & 1.2156 & 0.3532 & 0.2592 & 1.6163 \\
\hline Round 8 & 273.5545 & 0.9739 & 0.3823 & 0.2936 & 1.5508 \\
\hline Round 9 & 245.2390 & 1.5359 & 0.3011 & 0.2039 & 1.7756 \\
\hline Round 10 & 350.0889 & 2.2994 & 0.1892 & 0.1061 & 1.6990 \\
\hline
\end{tabular}

By result obtained by the AG - ACPT, the best fitness had a value of 1.3784, and occurred in the first round. For the parameters presented in this round the frequency of the particle, of $19.2392 \mathrm{MHz}$, and the resonance frequency of the cylindrical cavity, of $846.5230 \mathrm{MHz}$. Once again, the presence of bubbles in the equipment is made possible by a factor of 44 .

Fig. 7 shows the fitness evolution for the best condition presented by the AG - ACPT. It is verified that the best response occurred in the generation of number 65 as highlighted in the same figure, such condition occurred in the round of number 1 according to Table 6.

Figure 7: Evolution of fitness to the next 100 generations for the round that presented the best answer.

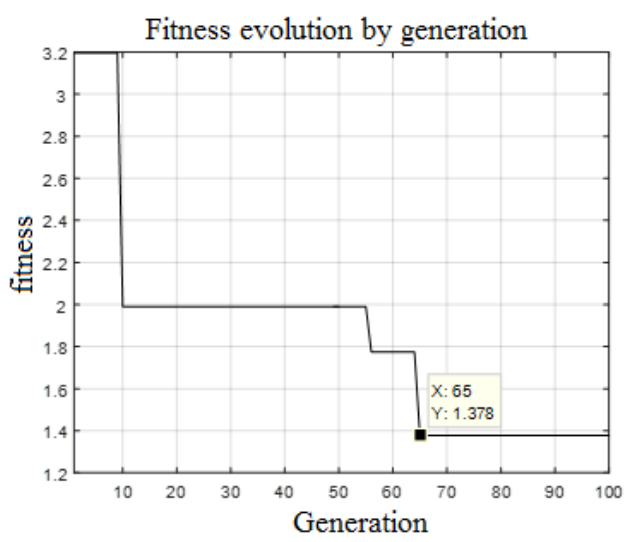

Source: by author. 
The parameters of the first round, presented as the best answer by the AG - ACPT, are highlighted in Table 7.

Table 7: Best response for the parameters for the circular particle accelerator according to the metaheuristic ACPT - 250.

\begin{tabular}{|c|c|}
\hline Potential difference (kV) & 339.9669 \\
\hline Magnetic field $(\mathrm{T})$ & 1.7060 \\
\hline Resonant cavity length $(\mathrm{m})$ & 0.2102 \\
\hline Resonant cavity radius $(\mathrm{m})$ & 0.1356 \\
\hline
\end{tabular}

Some important characteristics were found for the ACPT - 250 model, obtained with the help of the GA, as presented in the Table 8 .

Table 5: Features of the ACPT - 250.

\begin{tabular}{|c|c|}
\hline \multicolumn{2}{|c|}{ ACPT - 250 } \\
\hline Turns on accelerator & 720 \\
\hline Internal radius (m) & 0.1896 \\
\hline External radius (m) & 1.4248 \\
\hline Maximum Gap (m) & 0.7906 \\
\hline Frequency of accelerator cavity (MHz) & 846.523 \\
\hline Number of bubbles & 43.9999 \\
\hline Timing period (s) & $5.198 \times 10^{-8}$ \\
\hline
\end{tabular}

\section{CONCLUSION}

This paper presents the application of metaheuristics based on genetic algorithm to propose optimized parameters for circular particle accelerator model for proton beam radiation therapy, called ACPT. It is important to emphasize the proposal of two different devices, one focused on ocular therapy, ACPT -65 , and the other for chest therapy, ACPT - 250. Although it is important to highlight that such methodology can be applied to equipment with different purposes, the production of radioisotopes, as well as the burning of residues from nuclear processes, are excellent examples.

It is also important to mention that there are few papers that relate the topic of circular particle accelerators and genetic algorithms, or even evolutionary computation, so this work stands out for this use of GA. Based on studies such as (MUKHRJEE, 2000) and (TIAN; SAFRANEK; YAN, 2014), there 
is potential research in the application of evolutionary algorithms in the nuclear area, more specifically for particle accelerators, as a result of the recent development of genetic algorithms. However, it is also observed that they are researches for specific items present in particle accelerators. In this context, this pioneering work present values for some parameters of a circular particle accelerator, and its design is a direct result of the application of computational optimization, more precisely through genetic algorithms.

The development of the objective function that best represents the problem, as well as the use of the same restrictions are important issues that involved this work. It is also emphasized the importance in the employment and analysis of several generations for both computational simulations, since it became evident the evolution of the behavior of the GA in search of the best solution, evolving from generation to generation. Finally, it is believed that the AG - ACPT has great potential, as it presents specific parameters for each circular particle accelerator, dependent on its functional domain, associated with its energy level.

\section{REFERENCES}

WIESZCZYKA, W; SCHARF, W. H. "Proton Radiotherapy Accelerators." World Scientific P ublishing. 323 p. (2001).

MAGALLANES, L. HERNÁNDEZ; FERNÁNDEZ, A. Téllez. "Radioterapia con protones: la dosis precisa, ni más, ni menos.", 2015. Available at: <http://www.elementos.buap.mx/num99/ pdf/3.pdf>. Last accessed: 2017.

TUNER, S. Accelerator School. "Fifth General Accelerator Physics Course.” University of Jy väskylä, Finland, v. 1. Geneva, (1992).

NIFENECKER, H. et al. "Hybrid Nuclear Reactors." Progress in Particle and Nuclear Physic $s$, p. 683-827 (1999).

LOMAX, T. “An Overview of Compensated and Intensity Modulated Proton Therapy". PSI PROSCAN. AAPM Summer School, Colorado Springs, 7 p., 2003. Available at: < $\underline{w w w . a a p m . o}$ rg/meetings/03SS/.../Lomax.pdf>. Last accessed: 2017. 
MIVULE, Kato. "A Review of Genetic Algorithm", 2014. Available at: <https://www.slideshar e.net/kmivule/kato-mivule-geneticalgorithms1v1a>. Last accessed: 2017.

HOLLAND, J. “Adaptation in natural and artificial systems: an introductory analysis with ap plications to biology, control, and artificial intelligence." University of Michigan Press. ISBN 9 780472084609, 1975. Available at: <https://books.google.com.br/books?id=JE5RAAAAMAAJ >. Last accessed: 2017.

CARUANA, R. A.; Schaffer, J. D. "Representation and hidden bias: Gry vs. binary coding f or genetic algorithms.” In: Proceedings INTERNATIONAL CONFERENCE ON MACHINE L EARNING. Morgan Kaufmann, (1988).

MITCHELL, M. "An introduction to genetic algorithms." Cambridge: Mit Press. 207 p., (1997).

BANZHAF, W; NORDIN, P.; KELLER, R. E. \& FRANCONE, F. D. “Genetic Programming : an introduction.” ISBN 155860510X. Morgan Kaufmann, (1998).

POZO, Aurora; CAVALHEIRO, Andrea de Fatima; ISHIDA, Celso; SPINOSA, Eduardo; ROD RIGUES, Ernesto Malta. Computação evolutiva. Apostila do Departamento de Informática d a Universidade Federal do Paraná. Available at: <http://www.inf.ufpr.br/aurora/tutoriais/Ceap ostila.pdf>. Last accessed: 2016.

MUKHRJEE, Bhaskar. “Optimisation of the Radiation Shielding of Medical Cyclotrons usin g a Genetic Algorithm.” Japan Health Physics Society, Tokyo (Japan); 1 v; May 2000; [11 p.]; IRPA-10: 10. International Congress of the International Radiation Protection Association; Hiro shima (Japan); 14-19 May (2000).

TIAN, K.; SAFRANEK, Y.; YAN, Y. "Machine Based Optimization Using Genetic Algorith ms in a Storage Ring.” Physical Review Accelerators and Beams 17, 020703 - Published 18 Fe bruary (2014).

RABELO, Luísa de Araújo. "Projeto Eletromagnético de um Pós-Acelerador de Prótons para Tratamento de Tumor Ocular." Thesis of Doctorate in Nuclear Engineering at Universidade Federal de Minas Gerais. Belo Horizonte, (2016). 